\title{
Catalogs of celestial bodies from digitized photographic plates of the Ukrainian Virtual Observatory Archive
}

\author{
Protsyuk Y.*1, Vavilova I. ${ }^{\dagger 2}$, Andruk V², Pakuliak L. ${ }^{\ddagger 2}$, Shatokhina S. ${ }^{\S 2}$, and Yizhakevych O. ${ }^{2}$ \\ ${ }^{1}$ Research Institute 'Mykolaiv Astronomical Observatory', Mykolaiv, Ukraine \\ ${ }^{2}$ Main Astronomical Observatory of the NAS of Ukraine, Kyiv, Ukraine
}

\begin{abstract}
The Ukrainian Virtual Observatory (UkrVO, http://ukr-vo.org) database is compiled from observations conducted in 1898-2018 at observational sites of 8 Ukrainian observatories with about 50 instruments. Now the UkrVO archive covers data of about 40,000 astroplates, from which 15,000 are digitized at three observatories: Main Astronomical Observatory of the NAS of Ukraine (MAO NASU), Research Institute 'Mykolaiv Astronomical Observatory' (RI MAO), and Astronomical Observatory of the Taras Shevchenko National University of Kyiv (AO TSNU).

Astroplates were digitized using Epson ${ }^{T M}$ and Microtek ${ }^{T M}$ commercial scanners with 16bit gray levels and resolution of 1200-1600 dpi. Images of all objects registered on plates were processed using the advanced software complex for CCD images' treatment MIDAS/ROMAFOT in the LINUX environment. Additional software modules developed and implemented at the MAO NASU and RI MAO provide both the digitized images' processing and the final products as the catalogs of positions and stellar magnitudes of all the registered objects.

The processing of the digitized Northern Sky Survey observations (the FON project) resulted in a few catalogs of coordinates and B-magnitudes for more than 19 million stars and galaxies from the FON-Kyiv part and more than 13 million objects from the FON-Kitab part. Besides, based on these data, two catalogs for more than 5,000 positions and B-magnitudes of asteroids were compiled. Now, in cooperation with Hissar Astronomical Observatory (Dushanbe, Tajikistan), we are preparing similar catalogs based on digitized observations of the third part (1985-1992) of the FON project. The digitized data of open star clusters in UBVR color bands obtained at the Baldone observatory (Latvia) were used to enhance the photometric content of the resulted catalogs.

The developed methods of digitization, image processing, and reduction with the latest reference catalogs allowed us to achieve good positional and photometric accuracy of objects.

Other digitized data of photographic observations stored in UkrVO archives formed the basis of several Solar System Bodies positional catalogs. The compiled catalogs of 90 positions and B-values of Pluto, 1500 positions of satellites of outer planets are available on the UkrVO website and in the Strasbourg Data Center.
\end{abstract}

Keywords: Virtual Observatory, catalogs, database, stars, asteroids

\section{Introduction}

The Ukrainian Virtual Observatory (UkrVO, http://ukr-vo.org) database is compiled from observations conducted in 1898-2018 at observational sites of 8 Ukrainian observatories with about 50 instruments (Sergeeva et al. (2004), Protsyuk et al. (2005)). UkrVO database includes data from over 40 thousand direct astroplates, about 15 thousand of them have been digitized at three observatories and included in UkrVO Joint Digital Archive (UkrVO JDA): MAO NASU, RI MAO, and AO TSNU

\footnotetext{
*yuri@mao.nikolaev.ua, Corresponding author

†irivav@mao.kiev.ua

${ }^{\ddagger}$ pakuliak@mao.kiev.ua

§svetash@mao.kiev.ua
} 
(http://gua.db.ukr-vo.org/, http://nao.db.ukr-vo.org) (Vavilova et al. (2011), Vavilova et al. (2012), Vavilova (2016)).

Additionally, as an independent part of JDA, RI MAO hosts a digital archive of 140,000 CCD frames, having been obtained since 2001 with KT-50 $(\mathrm{D}=500 \mathrm{~mm}, \mathrm{~F}=3000 \mathrm{~mm}, 43$ 'x 38', scale $=$ $0.8^{\prime \prime} /$ pix $)$, FRT ( $\mathrm{D}=300 \mathrm{~mm}, \mathrm{~F}=1500 \mathrm{~mm}, 83^{\prime} \mathrm{x}$ 83'$\left.^{\prime}\right)$, and AMC ( $\mathrm{D}=180 \mathrm{~mm}, \mathrm{~F}=2480 / 12360$ $\mathrm{mm}$, scale $1 " / \mathrm{mkm}$ ) telescopes.

Besides direct plates' collections, UkrVO glass archive accumulates about 50,000 spectra astronegatives obtained in 1960-1995 (Pakuliak et al. (2014)), mostly with variable stars. At the moment, the project of their classification and digitization is in progress (Zolotukhina et al. (2020)).

The digitization of astroplates was carried out in all three observatories using $\operatorname{Epson}^{T M}$ and Microtek ${ }^{T M}$ commercial scanners with 16-bit gray levels, resolution of 1200-1600 dpi (Protsyuk et al. (2014b), Protsyuk et al. (2014c)) and stored in FITS format.

Digitization of astroplates and the newest digitized data processing services allowed us to develop a new approach for the creation of catalogs of astrometric and photometric characteristics of the various celestial objects. The accuracy of newly determined positions is higher than in traditional determinations from photographic observations, but it cannot be comparable to the accuracy of modern CCD observations. Modern observations far exceed the previous ones in the accuracy of coordinates and number of positions, but we hope to find from digitized photographic archives new original data about the Universe that could be useful in modern kinematic and dynamic solutions.

\section{Software for digitized images' processing}

Images of all objects registered on plates were retrieved using the advanced software complex for CCD images' processing MIDAS/ROMAFOT in the LINUX environment. Additional software modules developed and implemented at the MAO NASU and RI MAO provide both the object image extraction and the final products as the catalogs of positions and stellar magnitudes of all the registered objects. The detailed description of all steps of astronomical image processing is given in Protsyuk et al. (2014a), Protsyuk et al. (2014b), Andruk et al. (2014), Andruk et al. (2015), Pakuliak \& Andruk (2020). These steps are as follows: preliminary filtering, choosing PSF (point spread function) of objects, and the scheme of astrometric reduction to calculate positions and magnitudes in systems of the selected reference catalogs.

For all catalogs mentioned here, we used Tycho2 and UCAC4 catalogs as reference. Combined with developed methods of digitization, image processing, and plate reduction, these catalogs allowed us to achieve the best positional and photometric accuracy available for used photographic material and digitizing appliances. For example, mean internal errors of the FON-Kyiv resulted catalog (Tycho-2 as reference) are of 0.23 " in coordinates and $0.14^{m}$ in magnitudes for all stars down to $16^{m} .5$.

Using the Gaia DR2 as a reference showed that when compared with the results of the reduction in Tycho2 and UCAC4 reference frames, there was no significant improvement in the random and systematic components of residual differences $(O-C)$ for the EPSON scans from the UkrVO archive. On the other hand, the repeatability tests for the scans of the same plates obtained with the scanning machine of the Shanghai Astronomical Observatory (PRC) (Yu et al. (2017)) allow us to suppose that re-processing of old photographic observations in the Gaia DR2 reference system could improve the positional accuracy of old photographic observations, raising it to the level of modern ground-based CCD observations (Protsyuk \& Maigurova (2020)). It also can be assumed that these tests can be the indirect evidence of the advantages of the purpose-built machines against the flatbed scanners for scanning photographic plates.

Other UkrVO software and web-services are described in Vavilova et al. (2017). All software and web-services of the UkrVO are compatible with IVOA standards (Allen et al. (2019)).

\section{Catalogs of celestial bodies from digitized photographic plates}

The results of digitization, processing, and reduction are stellar catalogs with positional and photometric data for all objects fixed on the plates (Vavilova (2016), Vavilova et al. (2020)). Some catalogs 
can comprise proper motions of stars, which makes it possible to use them to obtain kinematic characteristics of objects and their groups. Since the start of the digitization of photographic archives of the UkrVO, we have created a set of catalogs of celestial bodies based on digitized images of photographic plates from the UkrVO JDA. Some of them are as follows:

- catalogs of coordinates and B-magnitudes for more than 19 million stars and galaxies from the Kyiv part and more than 13 million stars and galaxies from the Kitab part of the FON project (Andruk et al. (2016); Andruk et al. (2017); Yuldoshev et al. (2017); Yuldoshev et al. (2018); Yuldoshev et al. (2019));

- catalog of coordinates of 274485 stars (8-16 mag, Epoch J1981.6) from the observations of 19701990 using Zonal Astrograph of RI MAO (Protsyuk et al. (2014d));

- catalog of coordinates of 195 thousand stars down to $15^{m}$ in circumpolar areas (Protsyuk et al. (2015a));

- catalog of proper motions of 30 thousand stars down to $15^{m}$ in circumpolar areas (Protsyuk et al. (2015a));

- catalog of positions and proper motions of 2.3 million stars in the vicinity of open clusters NAO2015; (Protsyuk et al. (2014d); Protsyuk et al. (2017a));

- catalogs of more than 5,000 positions and B-magnitudes of asteroids (Shatokhina et al. (2018a); Shatokhina et al. (2018b); Shatokhina et al. (2019));

- catalog of 90 positions and B-magnitudes of Pluto; (Eglitis et al. (2019b));

- catalog of 1500 positions of satellites of Saturn, Jupiter, Uranus, and Neptune based on historical observations at MAO NAS of Ukraine. (Yizhakevych et al. (2017b); Yizhakevych et al. (2017a); Yizhakevych et al. (2018); Yizhakevych et al. (2019)).

Currently, in cooperation with Hissar Observatory (Dushanbe, Tajikistan), we are preparing similar catalogs based on the digitized observations of the third part (1985-1992) of the FON project. The digitized data of open star clusters in UBVR color bands obtained at the Baldone observatory (Latvia) were used to enhance the photometric content of the resulted catalogs.

The digital data from UkrVO JDA were used for the identification of optical counterparts of gamma-ray burst sources GRB110213A and GRB101224A. Catalogs of coordinates and magnitudes for all fixed faint objects in the areas around GRB110213A and GRB101224A were also created (Golovnia et al. (2015)). For these and other gamma-ray burst sources, the results were published in GCN Circulars Archive.

Re-processing of the digitized archive of Uranus and Neptune observations from RI MAO collection is carried out (Protsyuk et al. (2017b)).

The catalogs mentioned above cover both the whole northern hemisphere (the FON project) and its separate areas (near-polar, equatorial, local (open clusters, areas around GRB sources)). Observations carried out in different spectral bands are used.

\subsection{Catalogs of the FON project}

- Main idea for the FON project:

- FOUR-FOLD OVERLAP of the sky with instruments of the same type (Fig.1);

- SIX OBSERVATORIES: Kyiv(Ukraine), Kitab (Uzbekistan), Zelenchuk (Russia), Abastumani (Georgia), Zvenigorod (Russia), Dushanbe(Tajikistan);

- INSTRUMENTS: Double wide-angle astrographs, D/F=40/200, D/F=40/300;

- YEARS of observations: 1981-1998.

- Observational data for the compiled catalogs:

- FON-KYIV: 2260 plates, 24752709 stars and galaxies, epoch 1988.16, B color band, sky area from $-04^{\circ}$ to $+90^{\circ}$ on declination; 

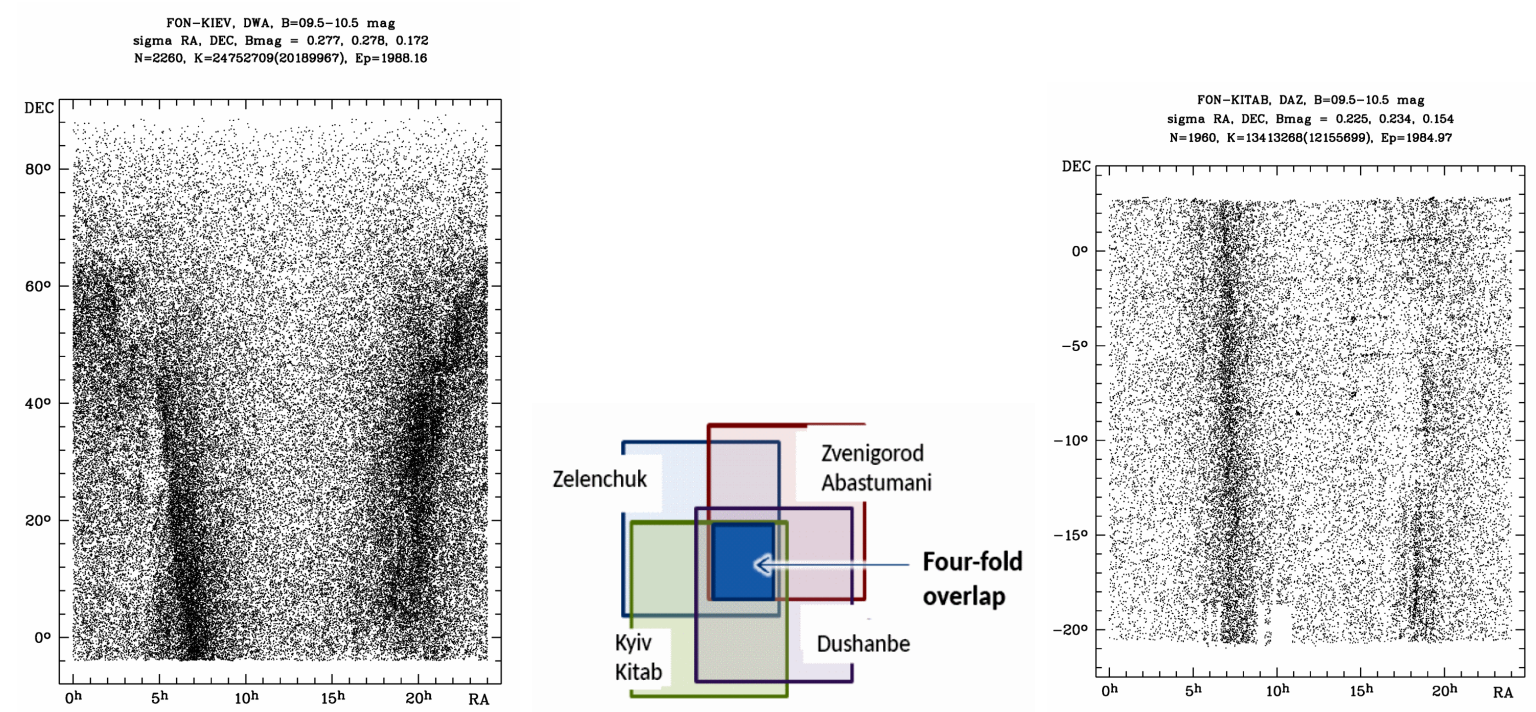

Figure 1. The scheme of the FON four-fold overlap (center). Bright part of the FON-KYIV catalog (left) and the FON-KITAB catalog (right).

- FON-KITAB: 1963 plates, 13413268 stars and galaxies, epoch 1984.97, B color band, sky area from $-20.5^{\circ}$ to $+2.5^{\circ}$ on declination;

- FON-DUSHANBE: 1560 plates, B color band, sky area from $-04^{\circ}$ to $+90^{\circ}$ on declination

- ADDITIONAL DATA:

* 1.2m Schmidt telescope, Baldone: 779 plates, U color band;

* 1.2m Schmidt telescope, Baldone : 4656 plates, V color band

- Results of the digitized images processing (Fig.1):

- FON-KYIV: declination zone: $-4.0^{\circ}$ to $+90.0^{\circ}, 24.7$ million objects, $\mathrm{B}<=16.5^{m}$, $\sigma_{R A, D E}= \pm 0.28^{\prime \prime}, \sigma_{B}= \pm 0.17^{m}$

- FON-KITAB: declination zone: $-20.5^{\circ}$ to $+2.5^{\circ}, 13.4$ million objects, $\mathrm{B}<=17.5^{\mathrm{m}}$, $\sigma_{R A, D E}= \pm 0.23^{\prime \prime}, \sigma_{B}= \pm 0.15^{m}$.

- FON-DUSHANBE: in progress, preliminary estimated $\sigma_{R A, D E}= \pm 0.33^{\prime \prime}, \sigma_{B}= \pm 0.12^{m}$.

FON-KYIV and FON-KITAB parts of the observational program are digitized, processed, and resulted in individual catalogs of positions and photometric data. The combination of digitizing technique with developed algorithms of scanner errors elimination and reduction models provided the accuracy of resulted data comparable to that of classical methods of photographic astrometry. The digitization of FON-DUSHANBE observational data makes it possible to create a final combined catalog of positions and proper motions of the Northern Sky, taking into account the main idea of the FON project - the multiple overlapping of the sky by several instruments of the same type (Akhmetov (2016); Akhmetov et al. (2018); Protsyuk \& Relke (2016)).

\subsection{Catalog of coordinates and proper motions from common reduction of CCD observation and plate archive images}

The combined processing of coordinates from digitized photographic (1953-1993) and modern CCD (1997-2020) observations has led to a new definition of the stars' proper motions. The VO world archives contain numerous original observational data in free access without any restrictions on their use. The possibility of downloading and processing files from these archives solves the problem of missing data. Based on CCD image files from various astronomical databases and joint processing of virtual and own CCD and photographic observations, the set of catalogs of positions and proper motions of stars was obtained in the areas of open star clusters (Fig.2). 

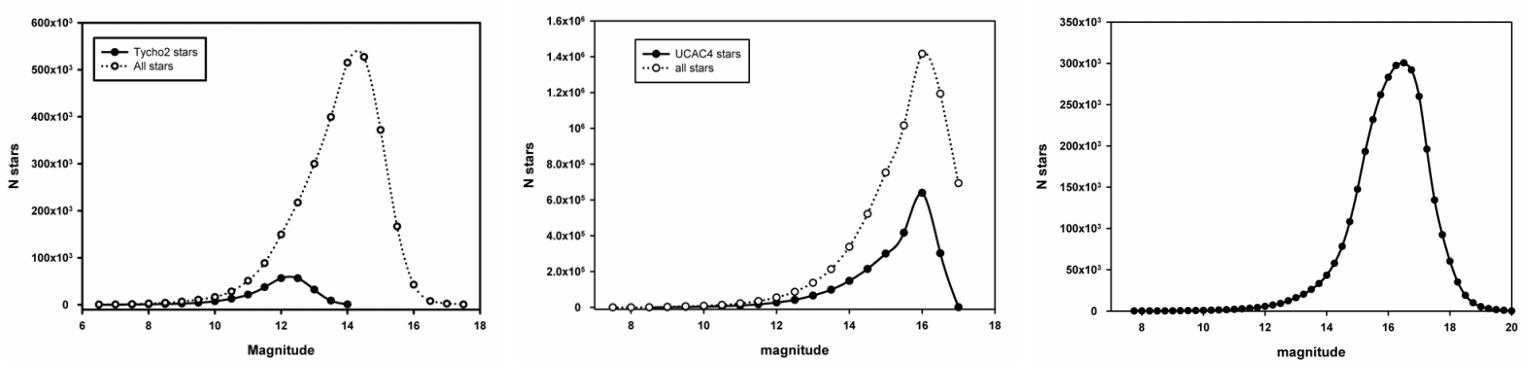

Figure 2. Distributions by magnitude of photographic catalog (left), CCD catalog (in the middle) and compiled catalog of positions and proper motions (right).

Catalogs which were obtained in 2012-2015:

- Photographic catalog for epoch 1983.2: $2.7 \mathrm{mln}$ stars $(7-16)^{m}, \sigma_{R A}= \pm 0.09^{\prime \prime}, \sigma_{D E}= \pm 0.10^{\prime \prime}$

- CCD catalog for epoch 2013.6: $4.2 \mathrm{mln}$ stars $(8-17)^{m}, \sigma_{R A}= \pm 0.06^{\prime \prime}, \sigma_{D E}= \pm 0.07^{\prime \prime}$

- 6 catalogs for different epochs from IVOA images: $51 \mathrm{mln}$ stars $(7-19)^{m}$, $\sigma_{R A, D E}= \pm(0.03-0.07)^{\prime \prime}$

* Catalog of coordinates and proper motions NAO2015: $2.3 \mathrm{mln}$ stars $(8-18)^{m}$, $\sigma_{R A}= \pm 0.035^{\prime \prime}, \sigma_{D E}= \pm 0.040^{\prime \prime}, \sigma_{\mu}= \pm 0.004^{\prime \prime} /$ year

The accuracy of the obtained coordinates and proper motions corresponds to the level of accuracy for modern ground-based CCD observations. The catalog also includes the stars of the open clusters of the Galaxy, which allows one to determine their stellar population and their average proper motions.

\subsection{Catalogs of asteroid positions and B-magnitudes from the FON-KYIV and FON-KITAB parts of the FON observational collections}

In addition to the star images, the massive photographic surveys of the sky comprise the records of moving objects of the Solar system. Extracting these data, we create some positional catalogs of asteroids, comets, outer planets and their satellites. Unlike the classical methods of image visualization, we used an analytical method to identify asteroids, based on a comparison of the obtained processing results and the calculated ephemeris of asteroids from on-line services.

We identified several thousand asteroids and derived 5020 their positions from the digitized observations of FON-KYIV and FON-KITAB collections. The preliminary catalog of about 1800 asteroid positions and UBVR-magnitudes was compiled in combination with the digitized observations from Baldone. Asteroids down to $16^{m}$ stellar magnitude were identified from the FON-KYIV part, down to $(17-17.5)^{m}$ from the FON-KITAB, and down to $18^{m}$ from the Baldone archive (Fig. 3). Comparison of all asteroid positions with ephemeris demonstrates a good agreement between the results obtained from the FON-KYIV and FON-KITAB digitized images.

Besides, mainly in the Baldone collection observed in 1967-1995, a large number of the faint asteroid precovery observations were found, and their positions and magnitudes were determined. The discovery of these objects took place only 20-30 years later, and they had no observations at all in the 20th century (Eglitis et al. (2019a)).

\section{Conclusion}

The external accuracy of star positions in our new photographic catalogs is in good agreement with their internal accuracy. The random positional error lies within 50 to 250 mas for most of them, which is due to the quality of the scanned material. Some of the catalogs, which comprise proper motions of stars, make it possible to use them to obtain kinematic characteristics of objects and their groups. We suppose that the usage of a high-precision reference catalog in the whole range of magnitudes 

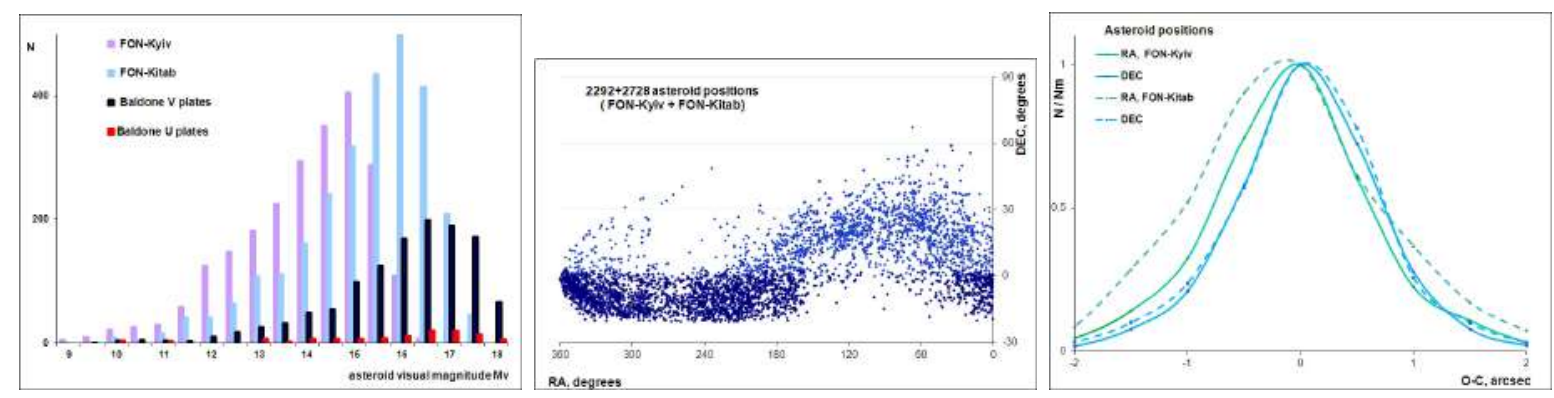

Figure 3. Distributions of asteroids positions by magnitude intervals (left), by equatorial coordinates (in the middle) and differences O-C (right), obtained from a comparison with ephemeris.

(for example, Gaia DR2) for the reduction will improve the star positions both systematically and randomly.

The use of new digital technologies for processing observations made it possible to increase the total number of positions of small bodies of the Solar System by searching for images from various digitized archives of observations, as well as to increase their accuracy. The combination of the Gaia DR2 (or DR3) stellar catalog with digital archives of the UkrVO gives a good possibility for data mining. As an example, the re-processing of some selected minor planets' observations using their digitized images and state-of-the-art star catalogs as a reference has led to increasing in positional accuracy compared to previous results (Protsyuk et al. (2016), Protsyuk \& Maigurova (2020)).

The covering of certain time intervals with missing data of asteroid positions and their analysis can be useful not only for modern ephemeris calculations but also for studying the evolution of asteroid orbits along time. The digital observational archives of UkrVO and other databases give a possibility to spot these missing data, retrieve them, and subject them to analysis (Savanevych et al. (2015), Savanevych et al. (2018)).

\section{References}

Akhmetov V. S., 2016, Odessa Astron. Publ., 29, 116

Akhmetov V. S., Khlamov S. V., Andruk V. M., et al. 2018, Odessa Astron. Publ., 31, 199

Allen M. A., Dowler P., Evans J. D., et al. 2019, arXiv e-prints, p. arXiv:1912.01481

Andruk V. N., Golovnya V. V., Ivanov G. A., et al. 2014, Odessa Astron. Publ., 27, 53

Andruk V. M., Pakuliak L. K., Golovnia V. V., et al. 2015, Odessa Astron. Publ., 28, 192

Andruk V. M., Pakuliak L. K., Golovnia V. V., et al. 2016, Kinem. Phys. Cel. Bod., 32, 260

Andruk V. M., Pakuliak L. K., Golovnia V. V., et al. 2017, VizieR Online Data Cat., I/342

Eglitis I., Yizhakevych O., Shatokhina S., et al. 2019a, Odessa Astron. Publ., 32, 189

Eglitis I., Eglite M., Kazantseva L. V., et al. 2019b, VizieR Online Data Cat., VI/155

Golovnia V., Protsyuk Y. I., Andruk V., et al. 2015, Odessa Astron. Publ., 28, 196

Pakuliak L., Andruk V., 2020, Applications of Big Data in Astronomy and Geosciences: Algorithms for Photographic Images Processing and Error Elimination. Škoda, P. and Adam, F., doi:10.1016/B9780-12-819154-5.00029-1

Pakuliak L., Shlyapnikov A., Rosenbush A., et al. 2014, in Astronomical Society of India Conference Series. H. P. Singh, P. Prugniel and I. Vauglin, pp 103-106

Protsyuk Y. I., Maigurova N. V., 2020, Odessa Astron. Publ., 33 
Protsyuk Y., Relke E., 2016, Odessa Astron. Publ., 29, 144

Protsyuk Y., Pinigin G., Shulga A., 2005, Kinematika i Fizika Nebesnykh Tel Supplement, 5, 580

Protsyuk Y. I., Andruk V. N., Kazantseva L. V., 2014a, Odessa Astron. Publ., 27, 59

Protsyuk Y. I., Andruk V. N., Muminov M. M., et al. 2014b, Odessa Astron. Publ., 27, 61

Protsyuk Y. I., Kovylianska O. E., Protsyuk S. V., et al. 2014c, Odessa Astron. Publ., 27, 63

Protsyuk Y. I., Martynov M. V., Mazhaev A. E., et al. 2014d, Kinem. Phys. Cel. Bod., 30, 296

Protsyuk Y., Andruk V., Mazhaev A., et al. 2015a, Odessa Astron. Publ., 28, 202

Protsyuk Y., Yizhakevych O., Kovylianska O., et al. 2015b, Odessa Astron. Publ., 28, 204

Protsyuk Y., Maigurova N., Protsyuk S., et al. 2016, Odessa Astron. Publ., 29, 147

Protsyuk Y. I., Kovalchuk O. M., Mazhaev O. E., 2017a, Sci. innov., 13, 54

Protsyuk Y. I., Kovylianska O. E., Protsyuk S. V., et al. 2017b, Sci. innov., 13, 89

Savanevych V. E., et al., 2015, Monthly Notices of the Royal Astronomical Society, 451, 3287

Savanevych V. E., et al., 2018, Astronomy\&Astrophysics, 609, A54

Sergeeva T. P., Sergeev A. V., Pakulyak L. K., Golovnya V. V., 2004, Baltic Astronomy, 13, 677

Shatokhina S., Relke H., Yuldoshev Q., et al. 2018a, Odessa Astron. Publ., 31, 235

Shatokhina S., Kazantseva L., Yizhakevych O., et al. 2018b, Kinem. Phys. Cel. Bod., 34, 270

Shatokhina S., Kazantseva L., Yizhakevych O., et al. 2019, VizieR Online Data Cat., J/other/KFNT/34

Vavilova I., 2016, Odessa Astron. Publ., 29, 109

Vavilova I. B., Pakuliak L. K., Protsyuk Y. I., et al. 2011, Kosm. nauka tehnol., 17, 74

Vavilova I. B., Pakulyak L. K., Shlyapnikov A. A., et al. 2012, Kinem. Phys. Cel. Bod., 28, 85

Vavilova I., Yatskiv Y., Pakuliak L., et al. 2017, in Astroinformatics. Brescia, M. and Djorgovski, S.G. and Feigelson, E.D. and Longo, G. and Cavuoti, S., pp 361-366, doi:10.1017/S1743921317001661

Vavilova I., Pakuliak L., Babyk I., et al. 2020, Surveys, Catalogues, Databases, and Archives of Astronomical Data. Škoda, P. and Adam, F., doi:10.1016/B978-0-12-819154-5.00015-1

Yizhakevych O., Andruk V., Pakuliak L., 2017a, Odessa Astron. Publ., 30, 201

Yizhakevych O. M., Andruk V. M., Pakuliak L. K., 2017b, Kinem. Phys. Cel. Bod., 33, 142

Yizhakevych O., Andruk V., Pakuliak L., 2018, VizieR Online Data Cat., J/other/KFNT/33

Yizhakevych E., Andruk V., Pakuliak L., 2019, VizieR Online Data Cat., J/other/OAP/29

Yu Y., Zhao J.-H., Tang Z.-H., et al. 2017, Research in Astronomy and Astrophysics, 17, 28

Yuldoshev Q. X., Ehgamberdiev S. A., Muminov M. M., et al. 2017, Kinem. Phys. Cel. Bod., 33, 250

Yuldoshev Q. X., Ehgamberdiev S. A., Muminov M. M., et al. 2018, VizieR Online Data Cat., I/346

Yuldoshev Q., Protsyuk Y., Relke H., et al. 2019, Astron. Nachr., 340, 494

Zolotukhina A. V., Pakuliak L. K., Vavilova I. B., Kaminsky B. M., 2020, Odessa Astron. Publ., 33 